\title{
Arbor
}

\section{Modelos educativos paradigmáticos en la Historia de la Educación}

\section{Isabel Gutiérrez Zuloaga}

Arbor CLXXIII, 681 (Septiembre 2002), 3-17 pp.

En este trabajo se analiza el proceso histórico de la Ciencia de la Educación, en cuanto que nos ayuda, no sólo a conocer y superar el pasado, sino también a iluminar el futuro. Para ello, se seleccionan algunas de las aportaciones histórico-educativas occidentales más influyentes desde el comienzo de la Edad Moderna. Son considerados como modelos paradigmáticos, a partir de la Edad Moderna: el Humanismo, el Realismo y el Naturalismo pedagógicos, así como la consideración científica de la Pedagogía; $y$ en el siglo XX: la Pedagogía sociológica, el Movimiento de la Escuela Nueva, el Personalismo pedagógico, la aplicación de la Tecnología a la enseñanza, y la propuesta europea de Educación ante el futuro. Se consideran sus principales aportaciones.

El proceso histórico de la Educación ha sido considerado por varios autores como una revisión evolutiva y perfeccionista del fenómeno pedagógico a través de los tiempos, de los países y de los grandes pensadores. Porque no podemos considerar el presente como algo absoluto, puesto que lleva en sí las virtualidades del pasado. Los problemas de origen, génesis y complejas influencias que plantea el mundo actual, sólo se hacen patentes a la luz de su consideración histórica.

Así lo entiende Herbart, para quien cada época trasmite a las siguientes sus pensamientos más elaborados, el tesoro de su lenguaje, y 
considera que en cada uno de nosotros vive el pasado por entero. Según Durkheim, la Ciencia de la Educación es precisamente un estudio de la evolución de los factores que, a través del tiempo, determinan el hecho educativo. Romagnosi afirma que el sujeto de la educación no es el hombre en cuanto individuo, sino en cuanto ser social, formado a través del desarrollo histórico de un pueblo. También en Estados Unidos se valora la visión histórica de la educación, como medio de explicar los complejos problemas que la Pedagogía se ha planteado a través de los siglos y de analizar sus soluciones.

Porque, si bien la Historia de la Educación nos sirve para conocer y superar el pasado, también nos ayuda a iluminar el futuro. Nos enseña a comprender el proceso pedagógico, para poder utilizar en su justo sentido las valoraciones esenciales y para adaptar los valores pasajeros a las concretas exigencias de cada momento histórico.

Por ello, nos hemos propuesto tratar las corrientes más destacadas que han influido en la Historia de la Educación y sus personajes-clave. Para lo cual, ha sido preciso realizar una selección de las aportaciones que pueden considerarse más significativas y de una mayor trascendencia posterior. Aunque, para ello, haya debido prescindir de otras propuestas que también considero de sumo interés.

De entre las aportaciones más influyentes que han dejado su huella en el proceso educativo occidental, a partir de la Edad Moderna -desde el siglo XVI hasta el siglo XIX inclusive- recordaremos las siguientes: el Humanismo pedagógico (siglo XVI); el Realismo pedagógico (siglo XVII); el Naturalismo pedagógico (siglo XVIII); la Sistematización científica de la Pedagogía (siglo XIX). Y del siglo XX destacamos: la Pedagogía sociológica; el Movimiento de la Escuela Nueva; el Personalismo pedagógico; la Tecnología de la Enseñanza; y la propuesta europea de la Educación ante el futuro.

\section{El Humanismo pedagógico}

La orientación sobre educación y enseñanza dada en el siglo XVI, siglo del Renacimiento y del Humanismo, está caracterizada -ante todopor su enfoque humanista, siendo su figura más representativa el valenciano Juan Luis Vives (1492-1540) quien, siendo nombrado profesor de la Universidad de Lovaina, instala su residencia en Brujas (Bélgica). Su obra pedagógica principal es el Tratado de la enseñanza, en cuya segunda parte, titulada «Sobre las disciplinas», aporta todo un programa para restaurar los estudios. 
Señala como objetivo de los estudios el hacer al joven más instruido y mejor. Expone la conveniencia de examinar las dotes mentales de los alumnos, y cómo los maestros deben observar y deliberar entre sí -en meses alternos y aún cada tres- sobre las cualidades de éstos, para poder orientarles con acierto y dedicarles a aquello para lo que demuestren más aptitudes.

Aunque admite que, para deducir el ingenio del alumno, no basta la simple y esporádica observación del profesor. Se precisa también, ponerle en ocasión de actuar y comprobar los resultados. Para realizar tales pruebas son útiles, tanto las matemáticas como el juego. Sobre el resultado de dichas técnicas exploratorias fundamenta Vives su división de los ingenios.

Su concepto de centro de enseñanza es muy amplio, ya que solicita la instrucción para todos los seres humanos. Piensa Vives (1923, p. 49) que debe constituirse la «verdadera Universidad» como un encuentro o convenio de personas doctas y buenas «reunidas para hacer iguales a ellas a cuantos acudiesen allí para aprender».

Defiende también la formación del gobernante y la de la mujer. En su tratado sobre La educación de la mujer cristiana destaca la importancia de la formación de la doncella, y señala entre sus fines el de la «adquisición de la cultura».

Watson ha calificado a Vives como padre de la Psicología moderna. Además, ha ejercido gran influencia en la Pedagogía posterior. Y quizás, lo que más ha trascendido de su obra haya sido, no tanto su parte teórica -sino más bien- la acertada aplicación de su teoría a los problemas pedagógicos.

\section{El Realismo pedagógico}

La gran figura del Realismo pedagógico en el siglo XVII es Juan Amós Comenio. Natural de Niewniz (Moravia), (1592-1670). Nos ha legado una gran obra, la Didáctica Magna, en la que ofrece como ideal la Pansofía o saber universal.

Esta Gran Didáctica, destacado ensayo pedagógico, parte de este principio fundamental: un confiado optimismo sobre la perfectibilidad del género humano, y sobre el poder de la educación para mejorar al hombre y a la sociedad. Concibe toda la realidad desde el punto de vista de la perfección humana. No trata sólo de la educación familiar y escolar, sino también de la solidaridad social y de la realidad natural. Ambas -naturaleza y sociedad-deben contribuir a la superación humana.

Comenio propone la elaboración de una sabiduría universal capaz de abarcar toda clase de conocimientos, reducidos a sus principios funda- 
mentales. Esta pansofía será la panacea que ha de apresurar el progreso moral, intelectual y espiritual de toda la sociedad. Dicha Ciencia universal debe darse a conocer en diversas etapas; en cada etapa se debe comunicar un conjunto completo de conocimientos, que será cada vez más elevado, profundo y extenso.

Propone que la educación alcance a todos los hombres. Todos deben acceder a la instrucción, que los capacita para su perfección y para su felicidad. Porque considera que la inquietud y la violencia que se dan en el mundo tienen por causa la desigualdad en la educación.

El primer ejercicio intelectual debe ser la observación de las cosas reales y sensibles, porque sólo los sentidos nos pueden dar a conocer las obras de la naturaleza. Deben ser los objetos, las cosas mismas, las que aparezcan tal como son ante la mente del discípulo. Por eso, el maestro debe procurar que el alumno se encuentre directamente con la naturaleza, ya porque ésta se trae a la escuela, o porque se utilicen imágenes que muestren lo que se pretende enseñar. Y para que resulte eficaz este contacto del alumno con la naturaleza, debe aplicarse el principio del orden; orden, no sólo en la distribución de la enseñanza, sino también en la presentación de las cosas.

En cuanto a la organización escolar, piensa que la enseñanza debe distribuirse en cuatro grados, según la edad de los alumnos y su programa de estudios, y que cada etapa debe durar seis años. Para el segundo grado - escuela elemental- señala como metas: el cultivo de la inteligencia, la imaginación y la memoria. El Gimnasio -tercer grado- es el adecuado para los ciudadanos que van a ejercer una profesión liberal. El cuarto grado corresponde a la Universidad. Propone también la fundación de un Colegio didáctico, dedicado al cultivo de las Ciencias y a fomentar el progreso de toda clase de descubrimientos que puedan servir para mejorar la vida humana.

Comenio es considerado como el padre de la Pedagogía moderna y el organizador y propagador de la escuela nacional. Porque acierta a integrar en su obra ideas de pedagogos anteriores a él y elabora un plan pedagógico de gran influencia posterior. Promueve formas educativas que hoy conservan su vigencia, como el empleo de la imagen, y da forma a una didáctica basada en los conceptos de naturalidad, intuición y autoactividad. De él nos dice A. Heyberger (1928) que «este educador... anima su obra con todos los sentimientos sublimes de un alma consagrada al servicio de la humanidad».

Su aportación pedagógica es muy destacada. En el siglo XIX se crea la «Fundación Comenio», y después aparece la «Sociedad Comenio» con carácter internacional. 


\section{El Naturalismo pedagógico}

Asignar a la naturaleza un papel decisivo y constituirla en ideal de la Humanidad, es el objetivo de Juan Jacobo Rousseau. Nace en Ginebra (Suiza), (1712-1778). Perteneciente al grupo de la Enciclopedia francesa, es uno de los mejores escritores del siglo XVIII; con él la Ilustración se supera a sí misma y da un paso más. Su obra pedagógica fundamental es el Emilio o sobre la educación, publicada en 1762. Rousseau hace de la naturaleza el fin y el método de la enseñanza. Propone la reacción a todo artificio, porque considera que se debe dejar hacer al alumno y que deben ser respetadas las etapas de su propio desarrollo.

El concepto rousseauniano de naturaleza humana hace referencia a todo aquello que es común a los seres humanos, de todos los lugares y de todos los tiempos. Supone, por tanto, una racionalización del concepto naturaleza. El Emilio presenta el ideal de hombre racional hacia el que debe tender toda educación: el hombre-naturaleza requiere una formación centrada en lo constitutivo de la naturaleza humana. Debe educarse, no para ejercer una determinada profesión u oficio, sino para ser verdaderamente hombre. Lo que realmente le interesa es la condición humana.

Una de sus principales aportaciones es el respeto a la naturaleza del niño, a la idiosincrasia infantil. Nos dice: «Dejad madurar la infancia en los niños». Solicita del educador que considere que el niño tiene maneras propias de ver, pensar y sentir, y que es insensato imponerle las nuestras.

La educación debe ser progresiva, teniendo en cuenta el movimiento evolutivo y gradual de la naturaleza infantil. Señala varias etapas: educación corporal o física, hasta los 2 años; educación sensorial, de los 2 a los 12; educación del espíritu, de los 12 a los 16; educación del corazón y la razón, de los 16 a los 20; cumplidos los 20 años, educación en la virtud y en la libertad moral. Progresión irreversible, porque cada etapa está montada sobre la anterior y prepara -a su vez- la siguiente.

Distingue Rousseau (1964, p. 89) lo que llama educación negativa, como opuesta a la educación positiva. Ésta es -para él- la que tiende a formar prematuramente el espíritu del niño y a instruirle en los deberes que corresponden al hombre. Educación negativa es la que tiende a perfeccionar los órganos, como instrumentos del conocimiento, antes de dar contenido alguno, y que procura preparar el camino a la razón, por el adecuado ejercicio de los sentidos. Tal concepto de educación negativa no supone mera pasividad del profesor, sino una vigilancia constante y oportuna, para ir preparando las situaciones propiamente educativas. No propugna la inactividad del educador, sino que le asigna una difícil mi- 


\section{Isabel Gutiérrez Zuloaga}

sión. Éste no debe imponer ninguna virtud, pero debe proteger del vicio; no debe inculcar la verdad, pero sí proteger del error. Debe preparar al niño para seguir el camino de la verdad, en cuanto llegue a la edad de comprenderla, y el camino de la bondad, cuanto pueda reconocerla y amarla.

Esta teoría rousseauniana de defensa de la humana naturaleza ha tenido una considerable influencia en la historia de la Educación. Entre sus principales seguidores contamos, ya en el siglo XVIII, con Pestalozzi en Suiza y con Basedow en Alemania. Ambos intentan plasmar sus ideas, no sólo en la teoría, sino también en instituciones de enseñanza, obteniendo un reconocido éxito.

\section{Sistematización científica de la Pedagogía}

La elevación de la Pedagogía al rango científico es un objetivo y un logro de Juan Federico Herbart. Éste nace en Oldenburg (Alemania), (1776-1841). Se propone investigar cuál es el principio fundamental del que parte la ciencia pedagógica, y lo encuentra en la moralidad, como fin mismo de la educación. Tal es el planteamiento que nos ofrece en su obra pedagógica más importante, titulada Pedagogía general derivada del fin de la educación, publicada en 1806.

Acierta a elevarse desde los aspectos empíricos e intuiciones dispersas, a los conceptos y a la sistematización lógica. Toma postura entre las dos perspectivas desde las que puede ser considerada la educación -ciencia o arte- para adoptar la primera, afirmando: nuestra esfera es la Ciencia.

$\mathrm{Su}$ actitud es realista, serena, equilibrada, racional. Según Ortega y Gasset, es una expresión coherente-desarrollada y evolucionada-del optimismo intelectualista de la Ilustración. Desde esta perspectiva, Herbart defiende que la instrucción tiene como misión el construir desde fuera y -por medio de estímulos externos- la personalidad del niño. Los dos pilares que sustentan y condicionan la Pedagogía son para él: la Psicología y la Ética. Ésta plantea unos fines y la Psicología proporciona los medios.

La misión del educador consiste -para él- en edificar el espíritu infantil, en construirle una experiencia determinada y claramente intuída; no en actuar como si el niño tuviese ya experiencia, sino cuidar de que obtenga esa experiencia. Y afirma que el educador cuenta, para cumplir su misión, con tres grandes instrumentos, que son: la instrucción, la cultura moral y el gobierno de los niños. 
Su idea de instrucción educativa está ligada al concepto de interés, que considera (Herbart 1935, pp. 60-61) el «concepto cardinal de la instrucción». Defenderá que la Pedagogía es la ciencia que necesita el educador para sí mismo. Pero -añade- que éste también debe conocer la ciencia para comunicarla. Porque no se puede concebir la educación sin la instrucción, y tampoco hay instrucción que no eduque. De aquí que el educador deba cuidar «la forma en que se establece el círculo de ideas en su discípulo, pues de éstas nacen los sentimientos, y de éstos los principios y modos de obrar».

El capítulo más difundido de su Didáctica es el de los grados formales de la educación. Estos pasos o grados son: claridad, asociación, sistematización y método. Su Pedagogía general es una importante aportación pedagógica porque, es a partir de su aparición cuando comienza a considerarse la Pedagogía como una Ciencia.

Entre sus numerosos discípulos, recordamos a T. Ziller, W. Rein y L. Strumpel.

\section{La Pedagogía sociológica}

Representante destacado de la Pedagogía que podemos denominar como sociológica es John Dewey. Natural de Burlington, Vermont (EE.UU.), (1859-1952). Dewey forma parte del movimiento de la «Educación nueva", movimiento que no se reduce a soluciones meramente didácticas, sino que ha contado también con pensadores teóricos, los cuales han logrado aportar a esta corriente serias bases de pensamiento. Así, dentro de esta línea, contamos con Dewey, principal teorizador de la «Educación nueva», en su doble línea de pedagogo de la acción y pedagogo de la experiencia. Destacamos entre sus obras, la publicada en el año 1916, que lleva por título Democracia y Educación.

Su reforma está fundada sobre la base del ideal educativo democrático. Los principios de su concepción ideológica se basan en tres tipos de revoluciones básicas: la de la ciencia, la de la industria y la de la social democracia. Esta última es la que -para él-supone una nueva y profunda concepción de la vida. Por eso afirma que han sido revolucionadas las condiciones radicales de la vida y que sólo una reforma pedagógica radical puede satisfacerlas completamente.

Propugna una socialización total de la educación y considera que, mediante la educación, debe desaparecer la distinción de clases. La escuela debe convertirse en una institución creada por la comunidad para mantener la vida y favorecer la prosperidad social. El sentido de la coopera- 
ción se despierta en el niño con la vida social. Los fines educativos tendrán su origen en las actividades reales de la vida, y serán tantos y tan variados como la vida misma. La experiencia educativa debe ser una constante reconstrucción de la actividad del niño.

En su obra Democracia y Educación deja claro su criterio de lo que debe ser la educación en una sociedad democrática. Se trata de una sociedad que procura la participación de todos sus miembros en términos iguales y que asegura un reajuste flexible de sus instituciones, mediante la interacción de las diferentes formas de vida asociada. Dicha sociedad debe contar con un modelo de educación que proporcione a los individuos un interés personal en las relaciones y el control social, y unos hábitos espirituales que aseguren los cambios sociales sin introducir el desorden.

Piensa que sólo una reforma pedagógica radical puede satisfacer el cambio en la concepción de la vida, y considera evidente que la relación entre democracia y educación sea «recíproca, mutua y vital». Ya que la propia democracia es un principio, una medida y un procedimiento educativos. Su práctica es un asunto educativo, porque pone ante nosotros la responsabilidad de considerar lo que deseamos como individuos.

También propugna Dewey (1933) un concepto pragmatista de la educación, cuando defiende el «enseñar por medio de la acción»-learning by doing- porque, según él, «sólo la acción manual y la intelectual promueven la experiencia», y la educación no es sino "una reconstrucción de la experiencia».

La «Escuela Dewey» de Chicago tiene como punto de partida las actividades del niño que - de modo natural y espontáneo- se producen en la casa y en la comunidad social en que se halla inserto. Porque, la escuela debe ser un instrumento adecuado para la especialización funcional de los intereses de los educandos. El maestro debe adaptar su actividad al estado actual del desarrollo del educando, tal como lo revelan sus intereses.

Varios pedagogos defenderán el ideal democrático y social propugnado por Dewey, quien es considerado como el principal representante de la defensa de una educación para la democracia.

\section{El movimiento de la Escuela nueva}

Entre los defensores de la Escuela Nueva, basados en la Psicología y el puerocentrismo, ocupa un destacado lugar María Montessori. Nace en Chiaravalle, Ancona (Italia), (1870-1952). Entre sus obras destaca El descubrimiento del niño, publicada en 1952. 
Montessori comienza trabajando con niños deficientes, lo que le lleva a reflexionar sobre la posibilidad de trasladar las conquistas de la Pedagogía de los subnormales y deficientes mentales, en medios para perfeccionar los métodos en la educación de la infancia normal. Para ello organiza -en un barrio de Roma- escuelas para niños en edad preescolar, que no habían podido ser atendidos por sus familias. Comienza así a funcionar la primera «Casa dei Bambini».

Su método se basa en su propio concepto del niño, como ser particular, cualitativamente distinto del adulto, dotado de maravillosas energías latentes que tienden al autodesarrollo. Piensa, además, que éste posee un amor puro y profundo, carente de dureza y de cálculo. Defiende que la humanidad, que se manifiesta ya con todos sus esplendores intelectuales en la tierna infancia, debe ser respetada con religiosa veneración.

En su obra El método de la pedagogía científica (Montessori 1932, pp. 92-93) defiende que el método pedagógico de la observación tiene por base la libertad del niño y que libertad ha de ser sinónimo de actividad. Llama disciplinado al individuo «que es dueño de sí y puede, por lo tanto, disponer de sí mismo cuando sea preciso». Y añade: «La libertad del niño debe tener como límite el interés colectivo; como forma de lo que llamamos la educación de las buenas maneras y de los actos». Defiende que la única acción educativa eficaz «será la que tienda a ayudar al completo desarrollo de la vida».

Sus postulados pedagógicos y su fe en el dinamismo de la infancia, se anticipan a la ciencia antropológica y pedagógica de nuestro tiempo. María Montessori ocupa actualmente un puesto privilegiado en la historia de la Educación infantil.

\section{El Personalismo pedagógico}

El pensamiento personalista es un pensamiento rebelde a una sistematización formal, a una unidad autoritaria. Su principal representante es Emmanuel Mounier. Nace en Francia (1905-1950). Su obra más destacada es El Personalismo, publicada en 1949.

Mounier define el personalismo como aquella doctrina que afirma la primacía de la persona humana sobre las condiciones materiales y sobre las estructuras colectivas que sostienen su desarrollo.

Para él, la persona es una unidad de vocación siempre capaz de superarse. Es la presencia y la unidad de una vocación intemporal, que urge a superarse indefinidamente a sí mismo y que opera, a través de la 
materia que la refleja, una unificación siempre imperfecta, siempre comenzada de nuevo. La primera misión de todo hombre consiste, pues, en descubrir de modo progresivo, esa cifra única que señala su sitio y su deber en la comunicación universal.

Son tres los ejercicios esenciales para la formación de la persona: la meditación, como búsqueda de la propia vocación; el compromiso, reconocimiento de su encarnación; y la depuración, como iniciación a la entrega de sí y a la vida en los demás. Cree que la persona sólo se encuentra dándose a la comunidad superior, que llama e integra a las personas singulares.

El personalismo se presenta como una pasión por el ser humano, y debe ofrecer líneas de conducta. La educación debe procurar una formación total de la persona humana. De aquí que los comentaristas de Mounier hayan destacado el carácter pedagógico de su pensamiento. Así, Paul Ricoeur (1950, pp. 862-863) nos dice que el personalismo es en su origen una pedagogía de la vida comunitaria, unida a un despertar de la persona. También Jean Lacroix califica a Mounier de educador.

En realidad, el personalismo supone en Mounier un esfuerzo para comprender y superar la crisis del siglo XX. Porque considera debe preparar la auténtica liberación del hombre. Dicho personalismo difundido por Mounier -desde 1932, a través de la revista Esprit- es defendido por muchos pedagogos actuales, que centran la atención educativa en los valores que autentifican a la persona humana. De ellos podemos recordar entre los pedagogos italianos, a L. Stefanini, M. Cassotti, G. Nosengo, G. Caló; entre los franceses, a J. Maritain; de los españoles, a J. Tusquets y a V. García Hoz.

\section{La Tecnología de la Enseñanza}

Se trata de utilizar las máquinas como técnica para aplicar los principios del aprendizaje. Figura pionera ha sido Burrhus Frèderic Skinner, profesor de la Universidad de Harvard. Nace en Dépot, Pensilvania (EE.UU.), (1904-1990). Se propone realizar una investigación del comportamiento, como hecho plenamente científico. Piensa que todo lo que podemos conocer en el campo del aprendizaje deriva del comportamiento, y que la mayor parte de la conducta y del aprendizaje humanos tiene un carácter operante. Aplica a dicho comportamiento operante la ley del refuerzo, entendida como resultado de un acontecimiento exterior que actúa sobre el organismo, fortaleciendo la conducta ante ciertos estímulos procedentes del ambiente. 
Utiliza Skinner (1958, p. 969) las máquinas como técnica para aplicar esos principios de aprendizaje. La máquina ofrecía la solución al problema de dar compensación inmediata -durante cada uno de los pasos- del estudio de un programa.

Piensa Skinner -en 1956- en una máquina que combinase las posibilidades de un computador electrónico con la modalidad de una respuesta escrita a máquina. Perfeccionando la idea, se van presentando máquinas que incluyen combinaciones audiovisuales.

Basándose en los trabajos experimentales de la escuela de Pressey, Skinner se propone realizar una investigación del comportamiento, considerado como hecho plenamente científico. Defiende que todo lo que podemos conocer en el campo del aprendizaje deriva del comportamiento. Aplica al comportamiento operante la ley del refuerzo, entendida como resultado de un acontecimiento exterior que actúa sobre el organismo, fortaleciendo la conducta ante ciertos estímulos procedentes del ambiente.

Un colaborador de Skinner, Holland, nos da la mejor definición de lo que puede llamarse técnica de programación de Skinner y sugiere (Fry 1962, p. 67) ocho reglas básicas para los programadores.

Skinner obtiene -en 1968- el Premio Nacional de la Ciencia. Es considerado como la figura contemporánea más polémica en el campo de la ciencia de la conducta humana.

\section{La Educación ante el futuro}

La Comisión Internacional para el desarrollo de la Educación, establecida por la Organización de las Naciones Unidas para la Educación, la Ciencia y la Cultura (UNESCO) publica -en 1972- una obra titulada Aprender a ser, cuya finalidad es llegar a soluciones de conjunto aplicables a las grandes cuestiones que plantea el desarrollo de la educación en un universo en devenir. El Presidente de dicha Comisión y uno de los autores del trabajo es Edgard Faure, nacido en Francia (1908-1988).

Faure escribe el «Preámbulo», en el que parte de la formulación de cuatro postulados (Faure 1972, p. 16): 1. La existencia de una comunidad internacional que se expresa por la comunidad de aspiraciones, de problemas y de tendencias; 2. La creencia en la democracia, concebida como el derecho de los hombres a realizarse plenamente; 3 . El desarrollo tiene por objeto el despliegue completo del hombre; 4. La educación debe ser global y permanente. Debe preparar al hombre para que elabore -a todo lo largo de su vida- un saber en constante evolución y de «aprender a ser». 


\section{Isabel Gutiérrez Zuloaga}

En la segunda parte del libro -titulada «Porvenires»- se intenta adivinar y preparar el progreso de la Educación. Se presentan como hechos portadores de porvenir los tres siguientes: 1 . las nuevas investigaciones de laboratorio en diversas ciencias; 2 . el desarrollo de la Pedagogía como ciencia y su relación con el ordenador, con el análisis de sistemas y con las nuevas tecnologías; 3. la modificación del papel de los enseñantes, del concepto de educación permanente y del proceso educativo global. En el capítulo denominado "Trascendencias» se intenta adivinar las consecuencias que traerán para la Educación los nuevos descubrimientos y cambios a nivel mundial.

La tercera parte -titulada «Hacia una ciudad educativa»- enfoca el camino abierto hacia el futuro, y finaliza con una propuesta de solidaridad.

La obra concluye con una seria propuesta por la mejora mundial de la Educación: «Proponemos que las instituciones de ayuda a la educación... examinen el estado presente de la «Investigación y Desarrollo» en materia de educación, con el fin de incrementar la capacidad de los diferentes países para mejorar su sistema educativo, concibiendo, organizando y comprobando experiencias educativas apropiadas a su cultura y a sus recursos». Y concluye (Faure 1972, p. 357) confiando en que «si se otorga prioridad... al reforzamiento de sus capacidades y sus medios, muchos países estarán en situación de dar los primeros pasos por el camino que conduce a la «Ciudad educativa».

Edgard Faure ha realizado grandes aportaciones en el campo educativo. Es también de interés la publicación -en 1968- de la obra titulada La educación nacional y la participación.

\section{Hacia una Ciudad educativa}

Recordemos los elementos educativos especiales que -a lo largo de la historia de la Educación- han aportado cada una de las corrientes seleccionadas en este estudio.

El humanismo pedagógico se propone hacer al joven más instruido y mejor. Solicita que el profesorado lleve a cabo -de modo periódico- una orientación escolar. Proclama una educación para todos, sin distinción de personas ni de edades. Defiende -en especial- la formación del gobernante y la formación de la mujer. Promueve una Pedagogía deferencial. Renueva los métodos de enseñanza y manifiesta la necesidad del contacto con la vida práctica.

El realismo pedagógico confía en el poder de la educación para mejorar al hombre y a la sociedad. Reclama una sabiduría universal como pa- 
nacea para el progreso moral, intelectual y espiritual de la sociedad. Defiende que todos los seres humanos deben acceder a la instrucción, y propaga la escuela nacional. Le preocupa el método, como medio más seguro para descubrir la realidad y para trasmitirla.

El naturalismo pedagógico defiende un nuevo enfoque de la naturaleza y un nuevo enfoque del ser humano y de la sociedad. El ideal se pone ahora en el concepto de «hombre natural». Trata de una «educación negativa», la cual consiste en procurar la perfección de los órganos, que son los instrumentos del conocimiento humano, antes de darles contenido alguno.

La sistematización científica de la Pedagogía, supone el intento de convertir en Ciencia la variada y compleja suma de elementos que contribuyen al fenómeno pedagógico. La Pedagogía se hace Ciencia y se diferencia claramente del arte de la educación. Porque, una Ciencia sólo se considera tal, cuando presenta su contenido debidamente sistematizado y fundamentado en principios teóricos y generales. El concepto cardinal de la instrucción será el interés.

La Pedagogía sociológica propugna una sociedad democrática, que procure la participación de todos sus miembros en términos iguales. $\mathrm{Pe}$ dagogía de la acción y de la experiencia, que estimula el interés personal en las relaciones y el control social.

El Movimiento de la «Escuela nueva» considera al niño cualitativamente distinto del adulto. Defiende el derecho a su libertad, admitiendo sólo como límite el interés colectivo. Nos presenta una variedad de métodos y de orientaciones.

El Personalismo pedagógico presenta a la persona humana como una realidad siempre capaz de superarse. Defiende los valores que hacen auténtica la realidad de la persona. Destaca la primacía de la persona sobre las condiciones materiales y las estructuras colectivas.

La Tecnología de la enseñanza aplica la Tecnología a la conducta y al aprendizaje humanos. Emplea la ley del refuerzo para fortalecer la conducta, ante ciertos estímulos procedentes del ambiente. Defiende la enseñanza programada.

Aprender a ser. La Educación del futuro es una publicación de la UNESCO, en la que se defiende la importancia del desarrollo humano, así como la necesidad de una educación permanente y de un proceso educativo global. Se confía en el desarrollo de la Pedagogía como Ciencia, su relación con el ordenador, con el análisis de sistemas y con las nuevas tecnologías. Propugna un humanismo científico, el desarrollo de la creatividad, el compromiso social y la formación del hombre completo. Propone a los países que se conduzcan por el camino que conduce a lo que denomina como «Ciudad educativa». 
Finalmente, queremos destacar que, así como en el proceso evolutivo de la Historia se observa el resultado de una continua búsqueda del hombre para resolver los problemas humanos, de igual modo, en el ámbito de la Educación resulta también evidente esa búsqueda continua y progresiva, esa inquisición permanente sobre cómo tratar a la naturaleza humana y sobre cómo mejorarla. De tal modo que la Pedagogía sea realmente un instrumento útil y eficaz para mejorar la vida personal y su actuación social.

\section{Bibliografía}

AzEvedo, F. de (1966): Sociología de la Educación. México: F.C.E.

Comenio, J. A. (1977): Didáctica Magna. México: Porrúa.

Comenio, J. A. (1992): Pampedia (Educación Universal). Madrid: U.N.E.D.

DALE, E. (1946): Audiovisual method in teaching. New York.

DEWEY, J. (1933): Pedagogía y Filosofía. Madrid: Beltrán.

DEWEY, J. (1967): Democracia y Educación. Buenos Aires: Losada.

FAURE, E. y otros (1973): Aprender a ser. La educación del futuro. Madrid: Alianza.

FRY, E. (1962): Máquinas de enseñar y enseñanza programada. Madrid: El Magisterio español.

García Hoz, V. (1970): Educación personalizada. Madrid: Instituto de Pedagogía del C.S.I.C.

Gutí́rReZ ZulOAGA, I. (1968): Historia de la Educación. Madrid: Iter Ed.

GuTIÉRREZ ZulOAGA, I. (1983): Diccionario de Ciencias de la Educación. Madrid: Ed. Rioduero.

GutiérRez Zuloaga, I. (coord.) (1990): Diccionario de Ciencias de la Educación. Madrid: Ed. Paulinas.

Helming, H. (1969): El sistema Montessori. Para un ejercicio de la libertad. Barcelona: Luis Miracle.

Herbart, J. F. (1935): Pedagogía general derivada del fin de la educación. Madrid: La Lectura.

HeyberGer, A. (1928): Jean Amos Comenio. Sa vie, son oeuvre d'éducation. París: Champion.

LUMSDAINE, A. A. (1965): Instrucción programada y máquinas de enseñar. Buenos Aires: Humanitas.

LUZURIAGA, L. (1961): Ideas pedagógicas del siblo XX. Buenos Aires: Losada.

MARÍN IBÁÑ̃z, R. (1972): Principios de la educación contemporánea. Madrid: Rialp.

Mataix, A. (1964): La norma moral en John Dewey. Madrid: Revista de Occidente.

MaYhew, K. y Edwars, A. C. (1936): La escuela Dewey. New York: Appleton-Century Company.

Morx, C. (1973): El pensamiento de Emmanuel Mounier. Barcelona: Ed. Estela.

MONTESSORI, M. (1932): El método de la Pedagogía científica. Barcelona: Araluce.

MONTESSORI, M. (1952): La scoperta del bambino. Garzanti.

MOUNIER, E. (1936): Manifieste Au service du personalisme. París: Montaigne.

MOUNIER, E. (1949): Le personalisme. París: Presses Universitaires de France. 


\section{Modelos educativos paradigmáticos}

MOUNIER, E. (1975): Revolución personalista y comunitaria. Madrid: Zyk.

RICOEUR, P. (1950): «Una filosofía personalista». En Esprit.

ROUSSEAU, J. J. (1964): Emilio o de la educación. Madrid: Edaf.

Skinner, B. F. (1958): «Teaching Machines». En Science and Human Behaviour, 128, $\mathrm{n}^{\circ}$ 3330.

SKINNER, B. F. (1968): Walden dos (Hacia una sociedad humana cientificamente construida). Barcelona: Fontanella.

SkinNer, B. F. (1976): Ciencia y conducta humanas. Barcelona: Fontanella.

TORRANCE, E.P. (1960): Talent and Education. Minnesota: University Press.

VIAL, J: (1979): La escuela rumbo al 2001. Madrid: Narcea.

VIVES, J. L. (1923): Tratado de la Enseñanza. Madrid: La Lectura. [Se recoge también en sus Obras Completas. Madrid: Aguilar, 1947]. 